\title{
Structure determination of human and murine $\beta$-defensins reveals structural conservation in the absence of significant sequence similarity
}

\author{
FINN BAUER, ${ }^{1}$ KRISTIAN SCHWEIMER, ${ }^{1,3}$ ENNO KLÜVER, ${ }^{2}$ \\ JOSE-RAMON CONEJO-GARCIA, ${ }^{2}$ WOLF-GEORG FORSSMANN, ${ }^{2}$ PAUL RÖSCH, ${ }^{1}$ \\ KNUT ADERMANN ${ }^{2}$ AND HEINRICH STICHT ${ }^{1}$ \\ ${ }^{1}$ Lehrstuhl für Biopolymere, Universität Bayreuth, D-95440 Bayreuth, Germany \\ ${ }^{2}$ IPF PharmaCeuticals GmbH, D-30625 Hannover, Germany
}

(Received June 19, 2001; Final Revision August 9, 2001; AcCePted August 9, 2001)

\begin{abstract}
Defensins are cationic and cysteine-rich peptides that play a crucial role in the host defense against microorganisms of many organisms by their capability to permeabilize bacterial membranes. The low sequence similarity among the members of the large mammalian $\beta$-defensin family suggests that their antimicrobial activity is largely independent of their primary structure. To investigate to what extent these defensins share a similar fold, the structures of the two human $\beta$-defensins, hBD- 1 and hBD-2, as well as those of two novel murine defensins, termed mBD-7 and mBD-8, were determined by nuclear magnetic resonance spectroscopy. All four defensins investigated share a striking similarity on the level of secondary and tertiary structure including the lack of a distinct hydrophobic core, suggesting that the fold is mainly stabilized by the presence of three disulfide bonds. In addition to the overall shape of the molecules, the ratio of solvent-exposed polar and hydrophobic side chains is also very similar among the four defensins investigated. It is significant that $\beta$-defensins do not exhibit a common pattern of charged and hydrophobic residues on the protein surface and that the $\beta$-defensin-specific fold appears to accommodate a wide range of different amino acids at most sequence positions. In addition to the implications for the mode of biological defensin actions, these findings are of particular interest because $\beta$-defensins have been suggested as lead compounds for the development of novel peptide antibiotics for the therapy of infectious diseases.
\end{abstract}

Keywords: $\beta$-defensin; NMR structure; peptide fold; antimicrobial peptide; chemotaxis; chemokine receptor

Antimicrobial peptides are important components of the innate immunity against microbial infections. They have been described in plants, tunicates, insects, fish, amphibia, and mammals, and are proposed to participate in the early host

Reprint requests to: Heinrich Sticht, Lehrstuhl für Biopolymere, Universität Bayreuth, Universitätsstraße 30, 95447 Bayreuth, Germany; email: Heinrich.Sticht@Uni-Bayreuth.de; fax: +49-921-553544.

${ }^{3}$ Present address: Novaspin Biotech GmbH, D-85748 Garching, Germany.

Article and publication are at http://www.proteinscience.org/cgi/doi/ $10.1101 / \mathrm{ps} .24401$. defense response against microorganisms. These peptides are usually cationic, and their mechanism of action generally involves membranolytic disruption, permeability, or pore formation against bacteria, fungi, and viruses (Vaara 1992; Boman 1995; Hancock et al. 1995). One of the major families of gene-encoded antimicrobial peptides found in mammals are the defensins-cationic peptides of 28-42 amino acids length containing three intramolecular disulfide bonds (Ganz and Lehrer 1995; Diamond and Bevins 1998; Bevins et al. 1999). Based on the pattern of cysteine connectivity, the mammalian defensins can be divided into two 
major subgroups termed $\alpha$ - and $\beta$-defensins. In $\alpha$-defensins, invariant disulfide bonds are formed in a 1-6, 2-4, and 3-5 order (Selsted and Harwig 1989), whereas $\beta$-defensins exhibit a 1-5, 2-4, and 3-6 cysteine pairing (Tang and Selsted 1993). Although disulfide linkages differ, the three-dimensional structure of both groups of peptides is very similar including an antiparallel $\beta$-sheet as a major element of secondary structure (Zimmermann et al. 1995).

The $\alpha$-defensins were among the first antimicrobial peptides discovered and are major constituents of the primary granules of mammalian granulocytes (Selsted et al. 1983, 1985). These peptides are also proposed to play a role in inflammation, wound repair, and specific immune responses (van Wetering et al. 1999).

The $\beta$-defensins were originally discovered in mammals as antimicrobial peptides of the airway epithelial cells (Selsted et al. 1993). In humans, an abundant $\beta$-defensin (hBD1) was initially discovered by analysis of large quantities of hemofiltrate (Bensch et al. 1995). hBD-1 mRNA is constitutively expressed in numerous tissues, including the gingiva, parotid gland, buccal mucosa, and tongue (Mathews et al. 1999; Sahasrabudhe et al. 2000). A second human $\beta$-defensin (hBD-2) is an inducible product of airway epithelia representing the dynamic component of the local epithelial defense system (Schröder and Harder 1999; O'Neil et al. 2000). Recently, a third human $\beta$-defensin, hBD-3, was isolated from skin (Harder et al. 2001). In general, each $\beta$-defensin exhibits a distinct spectrum of antimicrobial activity. $\beta$-Defensins are either constitutively expressed or induced upon stimulation by different inflammatory factors.
It was reported that both $\mathrm{hBD}-1$ and $\mathrm{hBD}-2$ bind and activate the chemokine receptor CCR-6, thus linking innate and adaptive immunity (Yang et al. 1999). A sequence alignment of mammalian $\beta$-defensins is shown in Figure 1.

Structural information about $\beta$-defensins is currently limited to the structures of bovine neutrophil $\beta$-defensin 12 (bBD-12; Zimmermann et al. 1995) and of hBD-2 (Hoover et al. 2000; Sawai et al. 2001). In addition, the solution structures of two defensin-like peptides (DLPs) from platypus venom have been determined (Torres et al. 1999, 2000) that share the $\beta$-defensin typical cysteine pattern and a similar tertiary fold but exhibit no antimicrobial activity. Because of the low number of three-dimensional structures available and the low sequence identity, it is not yet known which residues and which structural features are important for defensin function.

We have determined the solution structures of the human $\beta$-defensins hBD-1 and hBD-2 and of the two newly identified murine defensins mBD-7 and mBD-8, which were obtained by molecular cloning and sequence analysis, to investigate the degree of their structural similarity and to correlate their structure with the antimicrobial activity. In the case of hBD-1 and hBD-2 it was of additional interest to verify whether there are common structural principles of $\beta$-defensins and chemokines that might explain the binding of $\beta$-defensins to chemokine receptors. The structural characterization of the murine defensins $\mathrm{mBD}-7$ and $\mathrm{mBD}-8$ should provide information whether the defensin fold is influenced by the presence of two one-residue deletions compared with human defensins. Our approach started with

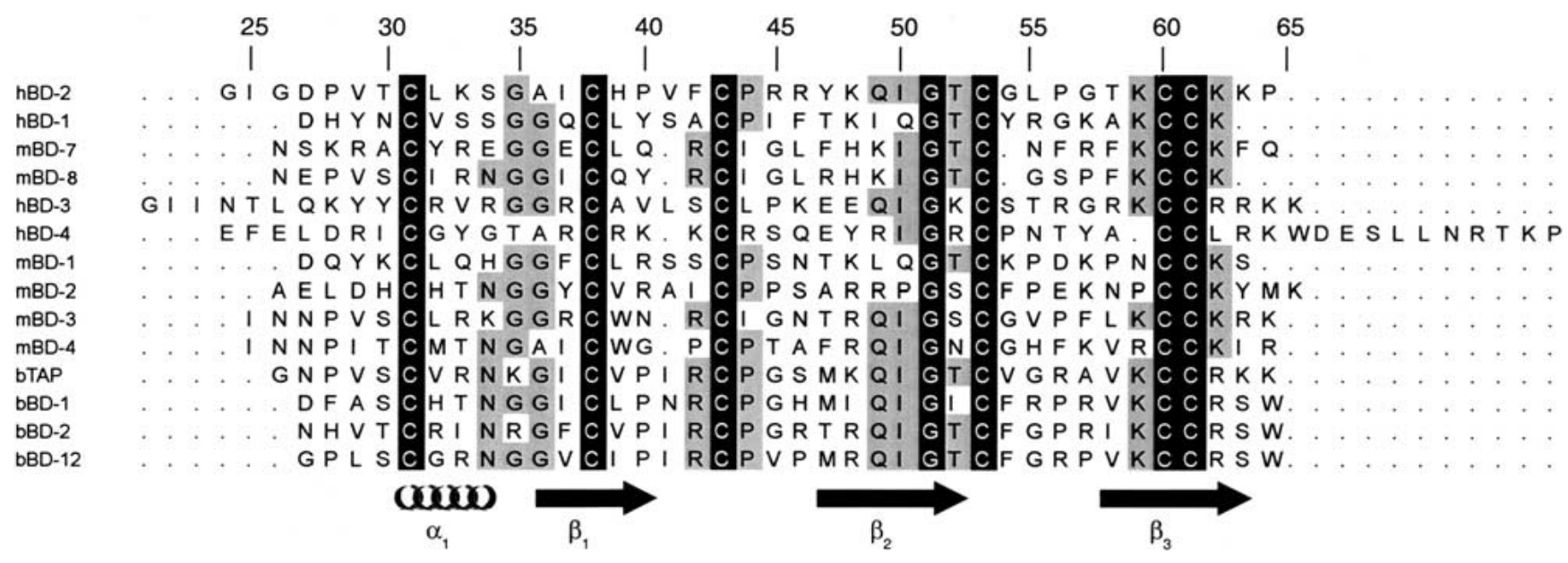

Fig. 1. Sequence alignment of mammalian $\beta$-defensins (BD). The alignment includes the sequences of four human defensins (hBD-1, hBD-2, hBD-3, hBD-4), six murine defensins (mBD-1 to mBD-4, mBD-7, mBD-8), three bovine defensins (bBD-1, bBD-2, bBD-12), and the bovine tracheal anitmicrobial peptide (bTAP). Strictly conserved amino acid residues are highlighted by a black box and residues occurring with a frequency of $\geq 50 \%$ are marked by gray boxes. The alignment was generated using the programs ClustalW (Higgins et al. 1992) and Alscript (Barton 1993). Because of the low sequence similarity, DALI analysis (Holm and Sander 1996) of the three-dimensional structures was used to allow a correct placement of the gaps in the sequences of mBD-7 and mBD-8. Elements of secondary structure found in hBD-1, hBD-2, mBD-7, and mBD-8 are schematically indicated below the alignment. The numbering scheme refers to the full-length hBD-2 including the amino-terminal signal sequence. 
the structure determination of hBD-2, which subsequently facilitated the structure determination of the three remaining defensins.

\section{Results and Discussion}

\section{Resonance assignments and secondary structure} of $h B D-2$

Homonuclear two-dimensional NMR-spectra of hBD-2 show a wide dispersion of amide and $\mathrm{C}_{\alpha}$-proton resonances from 7.28 to $9.89 \mathrm{ppm}$ and from 3.32 to $5.72 \mathrm{ppm}$, respectively. Sequence-specific resonance assignments were performed according to standard procedures (Wüthrich 1986) and allowed complete resonance assignment of all backbone and most of the side-chain protons.

Analysis of the nuclear Overhauser effect (NOE) pattern (Fig. 2) indicated the presence of three extended strands (residues 36-40, 47-52, and 58-63) forming a triplestranded antiparallel $\beta$-sheet and of a single helical turn for residues 31-34. Approximately 58\% of the long-range NOEs observed involved backbone protons indicating a lack of distinct side-chain packing interactions.

For residues 38 and 39 an additional minor conformation was detected exhibiting considerably upfield-shifted $(>0.4$ ppm) $\mathrm{H} \alpha$-resonances compared with the major conformation. This observation, together with the lack of the Cys 38-Cys $60 \mathrm{H} \alpha-\mathrm{H} \alpha \mathrm{NOE}$ indicates that this minor conformation does not form an extended $\beta$-strand for residues $37-40$, most probably because of the presence of a cisisomer for Pro 40. This hypothesis cannot be verified unambiguously because of frequency degeneration of the His 39-Pro40 $\mathrm{H} \alpha-\mathrm{H} \alpha$ NOE, but is supported by the lack of $\mathrm{HN}-\mathrm{H} \delta$ and $\mathrm{H} \alpha-\mathrm{H} \delta$ NOEs between His 39 and Pro 40, which are typical for a trans peptide bond. In contrast, the His 39-Pro 40 peptide bond of the major conformation as well as all remaining prolines were in the trans conformation as confirmed by strong sequential $\mathrm{H} \alpha-\mathrm{H} \delta$ NOEs to the preceding amino acid.

All of the NOEs observed for hBD-2 are consistent with the presence of a monomeric protein in solution. This finding is independently supported by the results from size exclusion chromatography, giving molecular masses of $\sim 5.7$ and $5.1 \mathrm{kD}$ for synthetic hBD-2 of 37 and 38 amino acid residues, respectively. From these reproduced results and in consideration of the accuracy of size exclusion chromatography it appears that the hBD-2 peptides investigated are monomeric in solution under the conditions tested.

\section{Tertiary structure of $h B D-2$}

The calculation of the final structures (Fig. 3) was based on 370 distance restraints, including 144 long-range NOEs (Table 1). The root mean square deviation (RMSD) of the
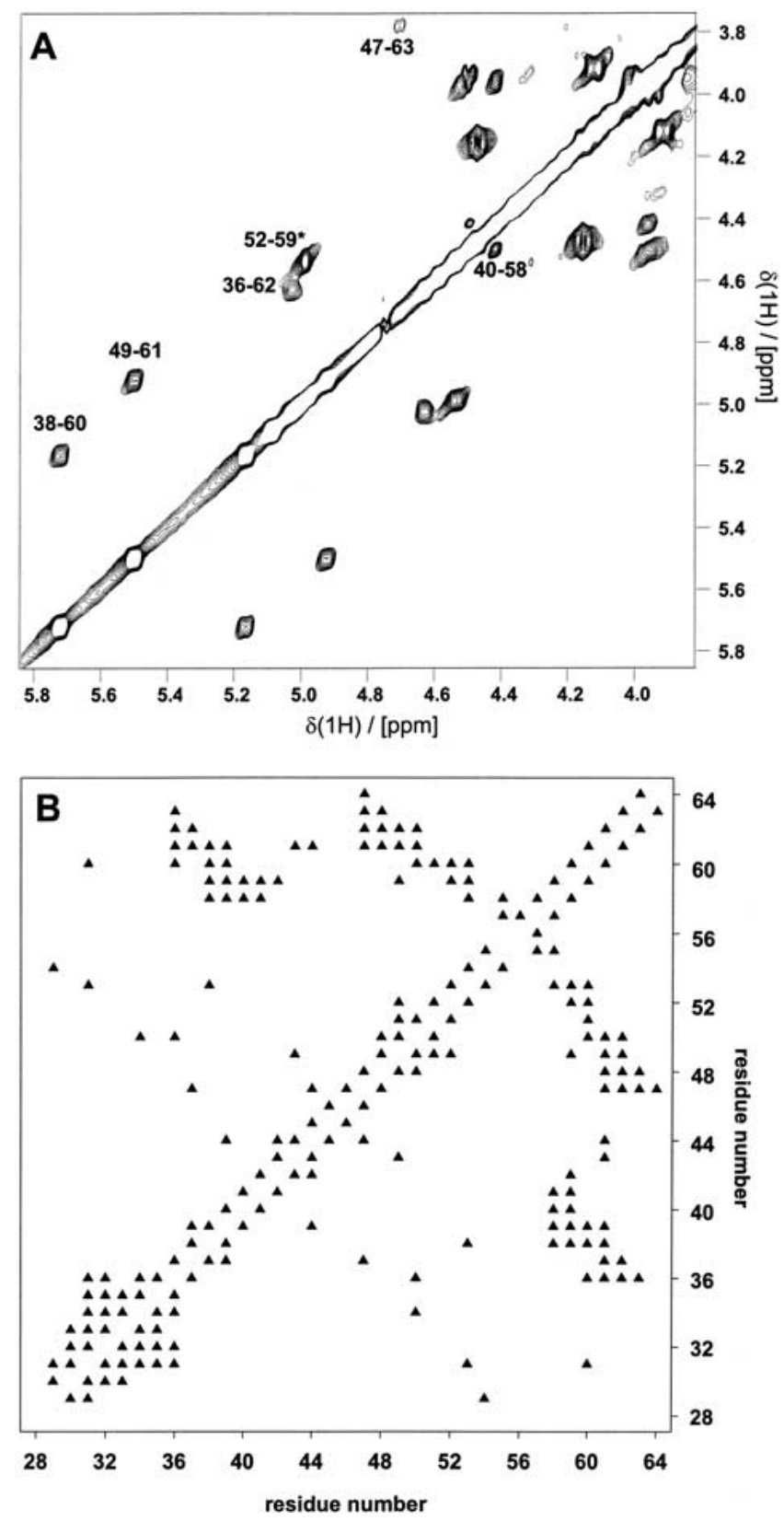

Fig. 2. Summary of the NMR spectroscopic data obtained for hBD-2. (A) Contour plot of the $\mathrm{H} \alpha-\mathrm{H} \alpha$ region of a $200 \mathrm{msec}$ mixing time NOESY spectrum of hBD-2 in $\mathrm{D}_{2} \mathrm{O}$. $\mathrm{H} \alpha-\mathrm{H} \alpha$ cross resonances indicative of $\beta$-strands are labeled. An asterisk denotes frequency degeneration of the corresponding cross-peak. (B) Summary of the spatial interactions between the amino acids in hBD-2. (A) NOEs that were observed between the corresponding residues. The length and position of the helix and $\beta$-sheets can be deduced from this presentation.

20 converging structures is $0.63 \AA$ for the backbone heavy atoms and $1.13 \AA$ for all heavy atoms. Omission of the terminal residues (28-30 and 62-64) from the calculation results in lower RMSD values of $0.50 \AA$ for the backbone heavy atoms and $1.09 \AA$ for all heavy atoms (Table 1; Fig. 4). 
A

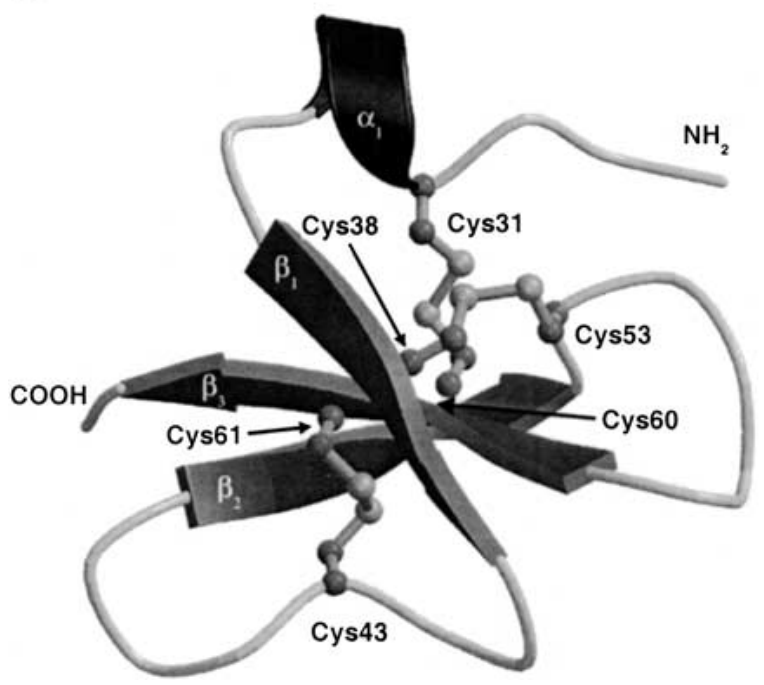

B

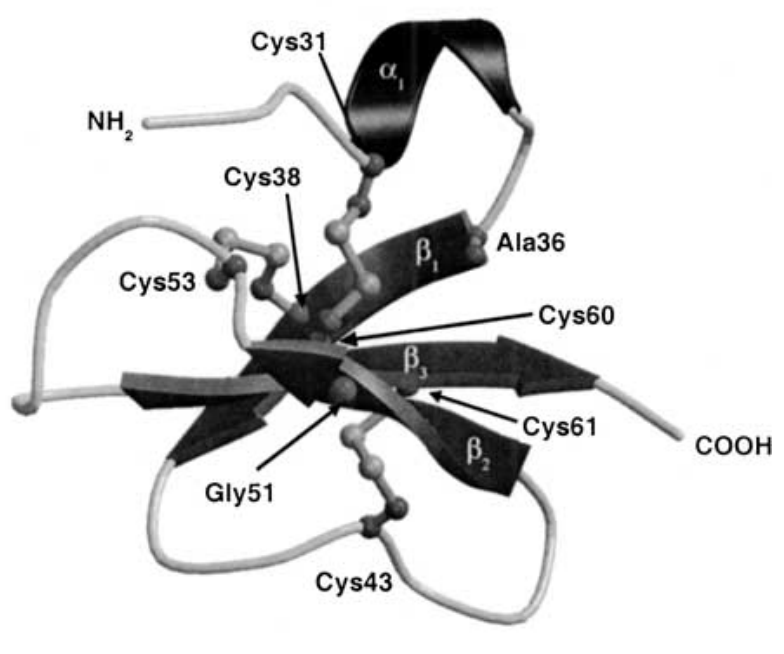

Fig. 3. Schematic representation of the solution structure of hBD-2. The elements of secondary structure and the disulfide bonds are indicated and highly conserved residues are shown in ball-and-stick representation. The two views in $A$ and $B$ are rotated by $\sim 180^{\circ}$ around the vertical axis. The figure was generated with Molscript (Kraulis 1991) and Raster 3D (Merritt and Murphy 1994).

According to PROCHECK analysis of the family of 20 structures, most residues show energetically favorable backbone conformations. $94 \%$ of the residues are found in the most favored regions and $2 \%$ in the allowed regions of the
Ramachandran plot. The unusual predominant backbone conformation of Val $41\left(\varphi \approx 60^{\circ}, \psi \approx-90^{\circ}\right)$ is identical to that of the corresponding isoleucine residue of bBD-12 (Zimmermann et al. 1995). This conformation most prob-

Table 1. Summary of the structure calculations

\begin{tabular}{|c|c|c|c|c|}
\hline Parameter & hBD-1 & hBD-2 & $\mathrm{mBD}-7$ & mBD-8 \\
\hline \multicolumn{5}{|l|}{ Average energy $(\mathrm{kcal} / \mathrm{mol})$} \\
\hline $\mathrm{E}_{\mathrm{tot}}$ & $34.9( \pm 0.3)$ & $60.1( \pm 1.1)$ & $40.7( \pm 1.0)$ & $49.5( \pm 1.5)$ \\
\hline $\mathrm{E}_{\mathrm{bond}}$ & $0.4( \pm 0.02)$ & $1.6( \pm 0.1)$ & $0.6( \pm 0.1)$ & $1.7( \pm 0.1)$ \\
\hline $\mathrm{E}_{\text {angles }}$ & $30.7( \pm 0.1)$ & $49.0( \pm 0.9)$ & $33.7( \pm 0.7)$ & $39.6( \pm 0.9)$ \\
\hline $\mathrm{E}_{\text {improper }}$ & $3.5( \pm 0.1)$ & $6.2( \pm 0.3)$ & $4.2( \pm 0.2)$ & $3.8( \pm 0.2)$ \\
\hline $\mathrm{E}_{\text {repel }}$ & $0.1( \pm 0.02)$ & $1.1( \pm 0.3)$ & $0.7( \pm 0.2)$ & $2.2( \pm 0.3)$ \\
\hline $\mathrm{E}_{\mathrm{NOE}}$ & $0.2( \pm 0.1)$ & $2.2( \pm 0.6)$ & $1.6( \pm 0.9)$ & $2.3( \pm 0.7)$ \\
\hline \multicolumn{5}{|c|}{ RMSD from ideal distances $(\AA)$ and angles (degree) } \\
\hline NOE & 0.005 & 0.011 & 0.011 & 0.016 \\
\hline bonds & 0.001 & 0.002 & 0.001 & 0.002 \\
\hline bond angles & 0.456 & 0.551 & 0.452 & 0.518 \\
\hline improper angles & 0.398 & 0.463 & 0.422 & 0.458 \\
\hline \multicolumn{5}{|l|}{ Ramachandran plot appearance } \\
\hline best & 89.5 & 93.9 & 85.0 & 96.9 \\
\hline favourable & 10.2 & 2.1 & 13.2 & 2.3 \\
\hline other allowed regions & 0.2 & 0.4 & 1.5 & 0.8 \\
\hline disallowed & 0.2 & 3.6 & 0.3 & 0.0 \\
\hline \multicolumn{5}{|l|}{ Atomic RMDSs } \\
\hline backbone (all residues) & 0.75 & 0.63 & 1.53 & 1.43 \\
\hline heavy atoms (all residues) & 1.31 & 1.13 & 2.25 & 1.93 \\
\hline backbone ("core fold") & 0.54 & 0.50 & 0.73 & 0.55 \\
\hline heavy atoms ("core fold") ${ }^{\mathrm{a}}$ & 1.11 & 1.09 & 1.55 & 1.22 \\
\hline \multicolumn{5}{|l|}{ Accessible surface area } \\
\hline$\%$ polar & 41.7 & 38.9 & 47.4 & 44.4 \\
\hline$\%$ hydrophobic & 58.3 & 61.1 & 52.6 & 55.6 \\
\hline
\end{tabular}

a The "core fold" was defined by the stretch of amino acids between the first and the last cysteine. 


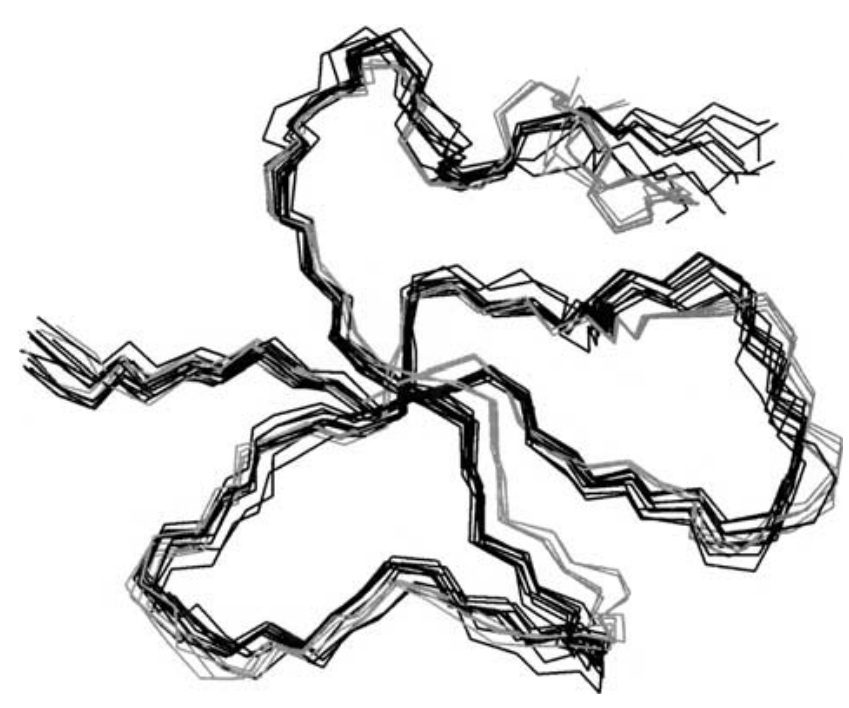

Fig. 4. Overlay of a set of 10 hBD-2 structures obtained by NMR spectroscopy (black) with 5 crystal structures (gray) from the work of Hoover et al. (2000) (pdb entries: 1FD3 and 1FD4). Same orientation as in Fig. 3a. Figure drawn with SYBYL 6.5.

ably correlates with the vicinity of a proline (Pro 40) in both defensins and is not observed in hBD-1 (see below) where the corresponding residue (Ser 41 ) is preceded by a tyrosine (Fig. 1).

Figure 3 shows a schematic presentation of the hBD-2 structure. The amino terminus containing the short helical turn is fixed by the Cys 31-Cys60 disulfide bond to the triple-stranded antiparallel $\beta$-sheet (Ala 36-Pro 40; Tyr 47Thr 52; Thr 58-Lys 63). Although the first and third strand of the $\beta$-sheet exhibit a regular geometry, a bend is present in the second strand. This irregularity correlates with the presence of an unusual backbone angle combination for residues Ile $50\left(\psi \approx-40^{\circ}\right)$ and Gly $51\left(\varphi \approx 160^{\circ}\right)$ in the majority of the structures calculated. As a consequence, the $\beta$-sheet is twisted around the 50-51 peptide bond, but the overall extended conformation remains unaffected.

The strands of the $\beta$-sheet are connected by two loops (Val 41-Arg 46; Cys 53-Gly 57) which show an increased conformational flexibility despite the presence of two disulfide bonds formed by cysteines 43 and 53 .

Analysis of the side-chain orientation confirms the conclusion from the NOE pattern. Most side chains (including most hydrophobic aliphatic and aromatic side chains) are exposed to the solvent, and there is no evidence for the presence of a hydrophobic core. For example, residues Leu 32, Val 41, Phe 42, and Leu 55 are $>40 \%$ solvent accessible.

\section{Comparison to the crystal structure of $h B D-2$}

Recently, a structure of hBD-2 was determined for two crystal forms that differed in the quaternary arrangement of the hBD-2 molecules (Hoover et al. 2000). In one form, a dimer was observed that is constituted by interactions between the first $\beta$-strand of each monomer resulting in a six-stranded $\beta$-sheet; the second crystal contained two octameric assemblies in the asymmetric unit, each composed of four hBD-2 dimers (Hoover et al. 2000). The high quality of the diffraction data allowed the independent structural description of 20 individual monomers, thus giving information about the conformational properties of hBD-2 in the crystal state (Hoover et al. 2000). From our NMR study, an ensemble of 20 solution structures is available providing the rare opportunity to compare two families of structures obtained by different experimental methods.

The pairwise $C \alpha-R M S D$ s for residues $31-62$ within each set of 20 structures obtained from X-ray and NMR data is in the range from $0.3-0.5 \AA$ and $0.5-0.8 \AA$, respectively. The higher RMSD values of the conformations in solution most likely reflect the increased conformational flexibility in this state compared with the crystalline state.

Comparison of the two sets of structures shows that the $\mathrm{C} \alpha$-RMSDs for residues $31-62$ are in the range from 1.1$1.4 \AA$. The major structural difference is observed for the conformation of the loops from residue 41-46 and from 53-57, which packs against the helical amino terminus in the crystal structure (Fig. 4). In the 37-residue hBD-2 peptide used for NMR investigation, the amino terminus is four residues shorter, resulting in a reduced number of interactions with the 53-57 loop compared with the crystal structure.

The helical amino terminus in the crystal structure also forms most of the intermonomeric interactions present in the oligomeric assemblies in both crystal forms. Despite this observation, there is experimental evidence that the length of the amino terminus alone is not the dominant factor determining the oligomerization state of hBD-2. Our results from size exclusion chromatography, giving a molecular weight of $3.9 \mathrm{kD}$, and static light scattering studies (Sawai et al. 2001) prove that the 41-mer is a monomer in solution up to millimolar concentrations suggesting that the oligomerization of the 41-mer in the crystal is mainly favored by the conditions used for crystallization including high protein concentrations of $>3 \mathrm{mM}$. This observation indicates that hBD-2 has the tendency to form oligomers under certain physiological conditions (e.g., high local concentration) and this tendency might have a role in particular defensin functions. It is therefore possible that $\beta$-defensins exert their antimicrobial effects as oligomeric molecules while they act monomeric on specific protein receptors.

Structures of the $\beta$-defensins $h B D-1, m B D-7$, and $m B D-8$, and comparison to $h B D-2$

To assess whether the structural features observed for hBD- 2 are also present in other $\beta$-defensins we determined 
the structures of the human defensin hBD-1 and of the new murine defensins mBD-7 and mBD-8 by NMR spectroscopy. Generally, the spectra of these defensins were of poorer quality than those of hBD-2. Nevertheless, >50 longrange NOEs could be assigned for each of these defensins allowing the determination of the overall fold. The structural statistics is summarized in Table 1.

All NOEs observed for the three peptides are consistent with the presence of monomeric defensins in solution. This finding is supported by the results of the size exclusion chromatography showing no peaks corresponding to molecular weights larger than $4 \mathrm{kD}$ and therefore no evidence for the existence of multimers.

Despite their low sequence similarity (Fig. 1) all four defensins investigated exhibit a virtually identical structure including a short amino-terminal $\alpha$-helix starting at the first cysteine residue and a triple stranded $\beta$-sheet (Fig. 5A-C). The length and relative arrangement of the three $\beta$-strands is also highly similar, including the characteristic twist in the second strand of the $\beta$-sheet correlated with an unusual backbone torsion angle combination for residues 50 and 51 (hBD-2 numbering scheme).

Interestingly, the position of the twist remains unaffected by the interchange of the relative positions of isoleucine and glutamate between hBD-2 (Glu 49-Ile 50) and hBD-1 (Ile 49-Glu 50), suggesting that the backbone conformation is determined by the defensin fold itself rather than by the sidechain properties of the amino acids at position 49 and 50. In contrast, Gly 51 is one of the few highly conserved residues in the defensin family (Fig. 1) suggesting that the predominantly observed backbone conformation in the lower right corner of the Ramachandran plot $\left(\varphi \approx 120^{\circ}\right.$ to
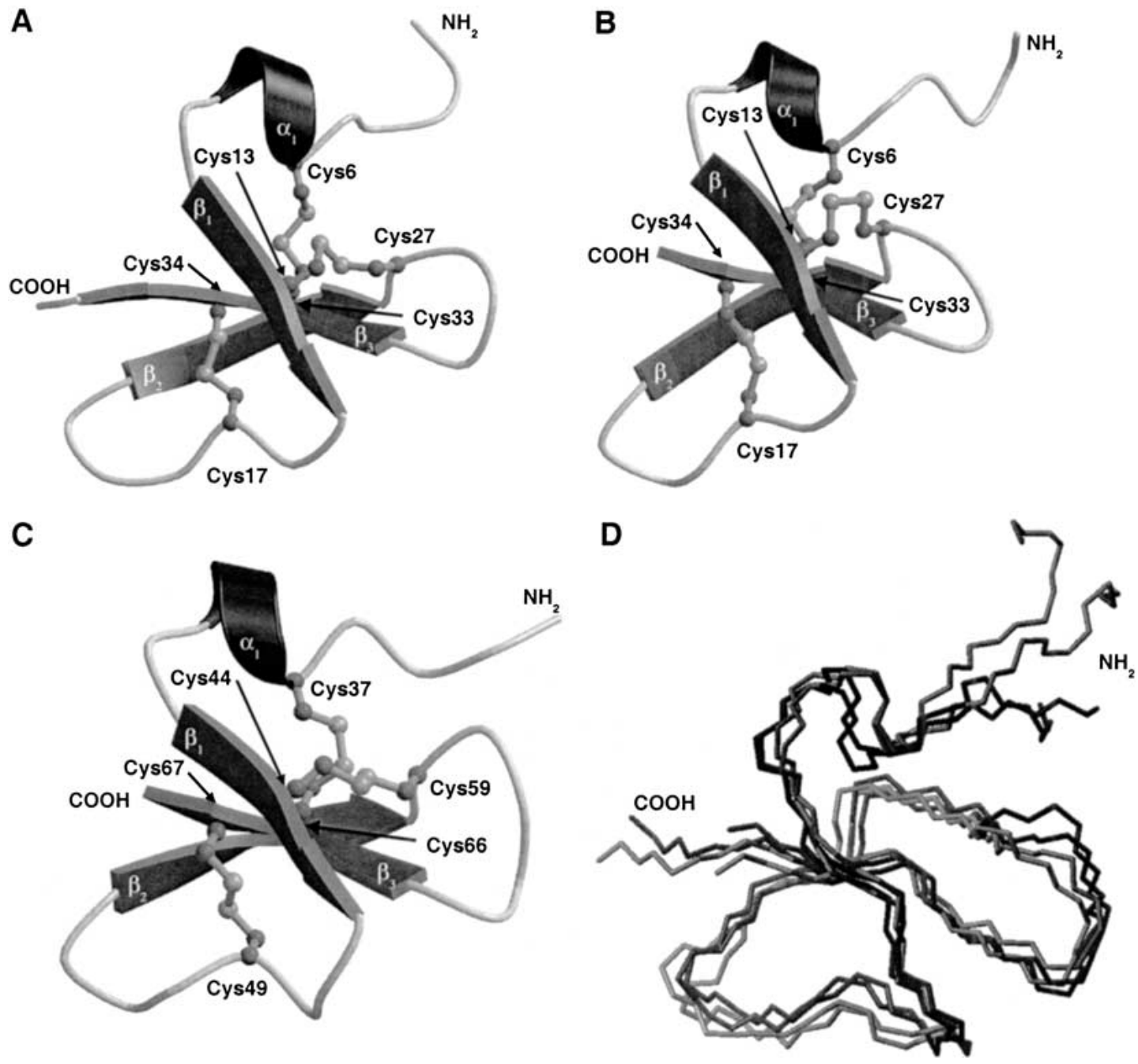

Fig. 5. Schematic representation of the solution structures of mBD-7 $(A), \operatorname{mBD}-8(B)$ and hBD-1 $(C)$. The elements of secondary structure and the disulfide bonds are indicated. Same orientation as in Fig. 3a. The figure was drawn with Molscript (Kraulis 1991) and Raster 3D (Merritt and Murphy 1994). (D) Overlay of the structures of the two human defensins hBD-1 and hBD-2 (dark gray) with the structures of the two murine defensins mBD-7 and mBD-8 (light gray); figure drawn with SYBYL 6.5. 
$180^{\circ}, \psi \approx-120^{\circ}$ to $-180^{\circ}$ ) can more easily be adopted by glycine compared with other amino acids.

The sequence alignment also reveals a second sequence position (no. 36 according to the hBD-2 numbering scheme) at which only the tiny amino acids glycine or alanine are tolerated in $\beta$-defensins (Fig. 1). A possible explanation for this preference comes from the plot of the solvent accessibility (Fig. 6) showing that residue 36 is highly buried in the interior of the molecule where a bulkier residue might be inconsistent with the defensin fold for steric reasons.

A more general impression about the overall similarity of all four defensins can be obtained from the overlay in Figure 5D and from the RMSD values obtained from a distance matrix alignment (DALI) search (Holm and Sander 1996). Both deletions present in the murine defensins (Fig. 1), which are located in the loop regions connecting the $\beta$-strands, have only minor influence on the local structure and do not affect the overall defensin fold (Fig. 5). This is consistent with the DALI analysis showing that 30 of the $\mathrm{C} \alpha$-atoms between the human and murine defensins are structurally equivalent, and $34 \mathrm{C} \alpha$-atoms were superimposable between the two human and between the two murine defensins, respectively.

The $\mathrm{C} \alpha$-RMSDs resulting from these pairwise comparisons of different defensins are in the range from 1.0-1.5 which is of the same magnitude as the RMSDs obtained between the NMR and the crystal structure of hBD-2. Therefore, it appears not valid to interpret these RMSDs in terms of real structural differences because they might at least partially reflect the limit of the accuracy that was achieved by the experimental techniques used here.

The presence of striking structural similarity despite a high sequence variability suggests that the structural prop-

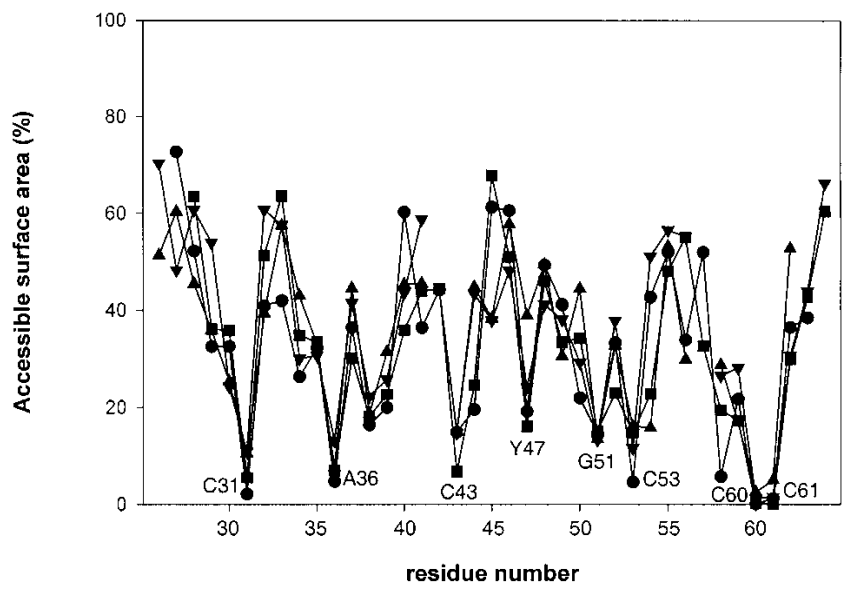

Fig. 6. Calculation of the accessible surface area per residue for hBD-1

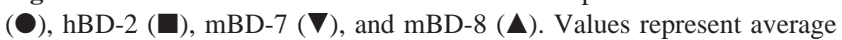
values for the 20 lowest-energy conformers of each defensin and were calculated using MolMol (Koradi et al. 1996). All sequence positions and those residues that exhibit the lowest solvent accessibility are labeled according to the hBD-2 numbering scheme. erties of the defensin fold are mainly stabilized by the three disulfide bonds. This is consistent with the observation that $\beta$-defensins lack a hydrophobic core necessary for stabilization of the tertiary structure in many other proteins (Julenius et al. 1998).

Calculation of the percentage of solvent accessibility per residue for all four defensins (Fig. 6) reveals that only a few amino acids are solvent-exposed by $<20 \%$ mainly including the six strictly conserved cysteines forming the disulfide bonds and two other highly conserved residues at sequence position 36 and 51. All other residues show a significant amount of solvent-exposed surface regardless of the physico-chemical properties of the corresponding amino acid sidechains.

Despite these common features and the presence of an overall similar shape of the protein surface, structure analysis failed to detect a common pattern for the distribution of polar and hydrophobic residues on the protein surface. For example, two of the most solvent-exposed hydrophobic residues in hBD-2 (Phe 42 and Leu 55) are replaced by alanine, arginine, asparagine, and glycine in the three other defensins investigated, whereas the two solvent-exposed arginines, Arg 45 and Arg 46, of hBD-2 are predominantly replaced by hydrophobic residues (Fig. 1). One common feature of all four defensins investigated, however, is the presence of five to eight positively charged residues resulting in quite similar (calculated) isoelectric points of 8.9 to 9.5. The portion of exposed hydrophobic groups is also quite similar for all four defensins, ranging from 53\%-61\%.

These findings suggest that the capability of $\beta$-defensins to interact with microbial membranes is determined by the shape of the molecules, the ratio of polar to hydrophobic residues on the peptide surface, or the total positive charge given through basic amino acids rather than by their individual primary structures. Minor difference of these structural features may be responsible for the specific antimicrobial spectrum, which is different for most $\beta$-defensins, and other important properties such as salt sensitivity and relative resistance towards protease degradation. Notably, the human $\beta$-defensin hBD-3 is highly salt-resistant (ConejoGarcia et al. 2001a; Harder et al. 2001) and kills the cystic fibrosis-related Burkholderia cepacia at an exceptionally low dose (Conejo-Garcia et al. 2001a), whereas the very recently described hBD-4 efficiently inhibits some strains of Pseudomonas aeruginosa, the major pathogen in cystic fibrosis (Conejo-Garcia et al. 2001b).

The distribution of charged residues was also proposed to play an important role for the ability of hBD-1 and hBD-2 to bind to the chemokine receptor CCR6 (Perez-Canadillas et al. 2001). The three-dimensional structure of macrophage inflammatory protein $3 \alpha$ (MIP-3 $\alpha$ ), the chemokine that specifically binds CCR6 (Baba et al. 1997; Krzysiek et al. 2000), was recently determined by NMR spectroscopy (Perez-Canadillas et al. 2001) and compared with that of 
hBD-2. The presence of a highly asymmetric distribution of positive charges in MIP- $3 \alpha$ and hBD-2 and the conservation of an aspartate and a leucine residue in the amino-terminal region of both proteins were suggested as factors that might contribute to the affinity and specificity of CCR6 receptor binding (Perez-Canadillas et al. 2001). Interestingly, these two features are also conserved in the structure of hBD-1 determined in our work. In hBD-1 an aspartate and a valine residue are present at those positions occupied by aspartate and leucine in hBD-2 and MIP-3 $\alpha$. In addition, hBD- 1 also shows a nonuniform distribution of positive charges resulting in two regions with an increased density of positive charges. The exact role of these structural features for the affinity and specificity of CCR6 binding, however, remains yet to be determined.

\section{Conclusions}

Accordingly, future results of particular significance can be expected from the identification and molecular analysis of further novel $\beta$-defensins and defensin-like peptides. The considerable sequence variability among $\beta$-defensins from a given species but also between different species suggests that the defensin fold is able to accommodate a wide range of amino acid residues at most sequence positions, not restricted to the naturally occurring derivatives. Based on the current knowledge of defensin structure, it is expected that extensive mutational studies will help to establish a structure-function relationship. This would contribute to unravel the mechanism of defensin action regarding the microbial cell wall and possible interactions with other microbial and mammalian proteins and to provide a molecular basis for the development of potential new drug therapies for the treatment of infectious diseases based on defensins.

\section{Materials and methods}

\section{Peptide synthesis}

mBD-7 and mBD-8 were identified by molecular cloning and subsequent sequence analysis. The corresponding nucleotide sequences have been deposited in GenBank with accession numbers $\mathrm{AJ} 298147$ and $\mathrm{AJ} 298148$ for $\mathrm{mBD}-7$, and $\mathrm{AJ} 300673$ and AJ300674 for mBD-8. Synthetic hBD-1, mBD-7, and mBD-8 were synthesized corresponding to the sequences shown in Figure 1, whereas hBD-2 was produced as two variants containing 37 and 38 residues differing in the amino-terminal length. Detailed conditions of the synthesis will be described elsewhere. Briefly, the peptides were assembled using standard Fmoc (fluorenylmethoxycarbonyl) chemistry on preloaded TentaGel S PHB or TentaGel S Trt resins (Rapp Polymere, Tübingen, Germany) on a 433A peptide synthesizer (Applied Biosystems, Weiterstadt, Germany) at a scale of 0.1 mmole yielding carboxy-terminal acids. The crude material was prepurified by C18 HPLC, and the reduced products were subjected to oxidative folding at basic $\mathrm{pH}$ in the presence of cysteine and cystine. Chromatographically homogenous products were obtained from these mixtures after purification by $\mathrm{C} 18$ HPLC. The molecular masses of the peptides were determined by ESIMS (hBD-1: $\mathrm{M}_{\mathrm{r}}$ calc $=3928.5, \mathrm{M}_{\mathrm{r}} \exp =3928.3 ; \mathrm{hBD}-2^{37}$ : $\mathrm{M}_{\mathrm{r}}$ calc $=3985.8, \mathrm{M}_{\mathrm{r}} \exp =3986.0 ; \mathrm{hBD}-2^{38}: \mathrm{M}_{\mathrm{r}}$ calc $=4100.9$, $\mathrm{M}_{\mathrm{r}} \exp =4099.9 ; \mathrm{mBD}-7: \mathrm{M}_{\mathrm{r}}$ calc $=4340.1, \mathrm{M}_{\mathrm{r}} \exp =4340.2$; mBD-8: $\mathrm{M}_{\mathrm{r}}$ calc $\left.=3836.5, \mathrm{M}_{\mathrm{r}} \exp =3836.9\right)$, and the amino acid sequences were confirmed by automated Edman degradation. The disulfide pattern was partially verified for mBD-7 and mBD-8, as basically described elsewhere (Conejo-Garcia et al. 2001b). Digestion of the peptides by trypsin and analysis of the resulting fragments by electrospray ionization mass spectrometry (ESIMS) and Edman degradation revealed a connection of the second and fourth cysteine, whereas the first and third cysteine are connected to the fragment containing the adjacent fifth and sixth cystein residue. Additionally, synthetic hBD-1 was shown to be identical with native hBD-1 (Bensch et al. 1995) by coelution and comigration upon HPLC and capillary electrophoresis, respectively.

\section{Size exclusion chromatography of $h B D-2$}

Three forms of hBD-2 differing in their amino-terminal length were investigated by size exclusion chromatography: (a) synthetic hBD-2 (37 amino acids, $\mathrm{M}_{\mathrm{r}}$ calc $=3985.8$ ); (b) synthetic hBD-2 (38 amino acids, $M_{r}$ calc $=4100.9$ ); and (c) recombinant hBD-2 (41 amino acids, $M_{r}$ calc $=4328.2$; Peprotech, Rocky Hill, NJ). Size exclusion chromatography experiments were also performed for hBD-1, mBD-7, and mBD-8 at concentrations in the range from $0.1 \mu \mathrm{g} / \mu \mathrm{L}$ to $1.0 \mu \mathrm{g} / \mu \mathrm{L}$. Each peptide was loaded on a Superdex 75 column (Pharmacia, Freiburg, Germany) equilibrated with $50 \mathrm{mM} \mathrm{K}_{2} \mathrm{HPO}_{4}$ buffer containing $0.1 \mathrm{M} \mathrm{Na}_{2} \mathrm{SO}_{4}$ (pH 7.35) using a flow rate of $40 \mu \mathrm{L} / \mathrm{min}$ and $\mathrm{UV}$ detection at 214, 230, and $280 \mathrm{~nm}$. The column was calibrated with a calibration standard, containing bovine serum albumin $(67 \mathrm{kD})$, ovalbumin $(43 \mathrm{kD})$, chymotrypsinogen A $(25 \mathrm{kD})$, ribonuclease A $(13.7 \mathrm{kD})$, and ubiquitin $(8.5 \mathrm{kD})$.

\section{NMR spectroscopy}

All defensin samples for NMR spectroscopy contained $\sim 1.0-2.5$ $\mathrm{mM}$ peptide in $0.5 \mathrm{~mL}$ of $\mathrm{H}_{2} \mathrm{O} / \mathrm{D}_{2} \mathrm{O}(9: 1$; vol/vol, $\mathrm{pH} 4.0)$ or $\mathrm{D}_{2} \mathrm{O}$ (99.994 atom \%). All NMR experiments were carried out at 400 and $600 \mathrm{MHz}\left({ }^{1} \mathrm{H}\right.$ frequency) on Bruker AMX400 and DRX600 spectrometers. For resonance assignment, correlated spectroscopy (COSY), total correlated spectroscopy (TOCSY), and nuclear Overhauser enhancement spectroscopy (NOESY) experiments were performed at $25^{\circ} \mathrm{C}$ using standard techniques for recording and water suppression (Cavanagh et al. 1996). TOCSY and NOESY experiments were recorded with mixing times of 40 and $80 \mathrm{msec}$, and 150, 200, and $300 \mathrm{msec}$, respectively. All experiments were acquired with 2048 complex points in $t_{2}$ and 256-512 complex points in $t_{1}$ and a sweep width of $12 \mathrm{ppm}$ in both dimensions. Data processing was performed using the Ndee software package (SpinUp Inc., Dortmund, Germany) on Sun and DEC workstations.

\section{Experimental restraints for the structure calculations}

NOE cross peaks were categorized as strong, medium, or weak and converted into upper limit distance constraints of 2.7, 3.5, and 5.0A, respectively. For distances involving either methylene protons without stereospecific assignments or methyl protons, single $\left\langle\mathrm{r}^{-6}\right\rangle^{-1 / 6}$ mean distances were used. Depending on the quality of 
the spectra obtained, 180-370 interresidual NOEs were used for the structure calculations including 50-144 long-range distance restraints observed between protons of amino acids more than four residues apart in sequence. One NOE distance restraint $\left(d_{\text {SS }}=2.02 \pm 0.10 \AA\right)$ was added for each of the three disulfide bonds. All structure calculations were performed iteratively, including only unambiguous distance restraints in the first rounds of the structure calculations.

\section{Structure calculations and analysis}

All structures were calculated using an ab initio simulated annealing protocol (Nilges et al. 1988) with a modified version of XPLOR 3.851 (Brünger 1993). The calculation strategy is similar to those described previously (Kharrat et al. 1995) and included floating assignment of prochiral groups (Holak et al. 1989), a conformational database potential term (Kuszewski et al. 1996), and a reduced presentation for non-bonded interactions for part of the calculation (Nilges 1993) to increase efficiency. The conformational search phase ( 45 psec of molecular dynamics at $2000 \mathrm{~K}$ ) was followed by a refinement in which the system was cooled from $2000 \mathrm{~K}$ to $1000 \mathrm{~K}$ within $20 \mathrm{psec}$, concomitantly increasing the force constants for the non-bonded interactions and the angle energy constant for the diastereospecifically unassigned groups to their final values. In the next stage of the calculation the system was cooled from $1000 \mathrm{~K}$ to $100 \mathrm{~K}$ within $20 \mathrm{psec}$, applying the high force constants obtained at the end of the previous cooling stage. To detect the energy minimum, 400 steps of energy minimization were performed, the final 200 steps without conformational database potential.

Of the 60 structures resulting from the final round of structure calculation, those 20 structures that showed the lowest energy and the least violation of the experimental data were selected for further characterization. The coordinates were deposited in the Protein Data Bank (codes: 1E4Q, 1E4R, 1E4S, 1E4T).

Geometry of the structures and elements of secondary structure were analyzed using PROCHECK (Laskowski et al. 1993) and solvent accessibilities were calculated with MolMol (Koradi et al. 1996). For the graphical presentation of the structures the programs SYBYL 6.5 (Tripos Ass., St. Louis, Missouri), RasMol (Sayle 1995), MOLSCRIPT (Kraulis 1991), and Raster3D (Merritt and Murphy 1994) were used.

\section{Acknowledgments}

This work was supported by the Mukoviszidose e.V. (to W.G.F.) and by the Fonds der Chemischen Industrie (to H.S.).

The publication costs of this article were defrayed in part by payment of page charges. This article must therefore be hereby marked "advertisement" in accordance with 18 USC section 1734 solely to indicate this fact.

\section{References}

Baba, M., Imai, T., Nishimura, M., Kakizaki, M., Takagi, S., Hieshima, K., Nomiyama, H., and Yoshie, O. 1997. Identification of CCR6, the specific receptor for a novel lymphocyte-directed CC chemokine LARC. J. Biol. Chem. 272: 14893-14898.

Barton, G.J. 1993. ALSCRIPT: A tool to format multiple sequence alignments. Protein Eng. 6: 37-40.

Bensch, K.W., Raida, M., Mägert, H.J., Schulz-Knappe, P., and Forssmann, W.G. 1995. hBD-1: A novel beta-defensin from human plasma. FEBS Lett. 368: $331-335$.
Bevins, C.L., Martin-Porter, E., and Ganz, T. 1999. Defensins and innate host defence of the gastrointestinal tract. Gut 45: 911-915.

Boman, H.G. 1995. Peptide antibiotics and their role in innate immunity. Annu. Rev. Immunol. 13: 61-92.

Brünger, A.T. 1993. X-PLOR Version 3.1. Howard Hughes Medical Institute \& Yale University, New Haven, CT.

Cavanagh, J., Fairbrother, W.J., Palmer III, A.G., and Skelton, N.J. 1996. Protein NMR Spectroscopy. Academic Press, San Diego.

Conejo-Garcia, J.R., Jaumann, F., Schulz, S., Krause, A., Rodriguez-Jimenez, F.J., Forssmann, U., Adermann, K., Klüver, E., Vogelmeier, C., Becker, D., Hedrich, R., Forssmann, W.G., and Bals, R. 2001a. Identification of a novel, multifunctional $\beta$-defensin (hBD-3) with specific antimicrobial activity: its interaction with plasma membranes of Xenopus oocytes and the induction of macrophage chemoattraction. Cell Tissue Res. (in press).

Conejo-Garcia, J.R., Krause, A., Schulz, S., Rodriguez-Jimenez, F.J., Klüver, E., Adermann, K., Bals, R., and Forssmann, W.G. 2001b. Human $\beta$-defen$\sin$ 4: A novel inducible peptide with a specific salt-sensitive spectrum of antimicrobial activity. FASEB J. 15: 1819-1821.

Diamond, G. and Bevins, C.L. 1998. $\beta$-defensins: Endogenous antibiotics of the innate host defense response. Clin. Immunol. Immunopath. 88: 221-225.

Ganz, T. and Lehrer, R.I. 1995. Defensins. Pharmacol. Ther. 66: 191-205.

Hancock, R.E., Falla, T., and Brown, M. 1995. Cationic bactericidal peptides. Adv. Microb. Physiol. 37: 135-175.

Harder, J., Bartels, J., Christophers, E., and Schröder, J.M. 2001. Isolation and characterization of human beta-defensin-3, a novel human inducible peptide antibiotic. J. Biol. Chem. 276: 5707-5713.

Higgins, D.G., Bleasby, A.J., and Fuchs R. 1992. CLUSTAL V: Improved software for multiple sequence alignment. Comput. Appl. Biosci. 8: 189191.

Holak, T.A., Nilges, M., and Oschkinat, H. 1989. Improved strategies for the determination of protein structures from NMR data: the solution structure of acyl carrrier protein. FEBS Lett. 242: 218-224

Holm, L. and Sander, C. 1996. Mapping the protein universe. Science 273: 595-603.

Hoover, D.M., Rajashankar, K.R., Blumenthal, R., Puri, A., Oppenheim, J.J., Chertov, O., and Lubkowski, J. 2000. The structure of human beta-defensin-2 shows evidence of higher order oligomerization. J. Biol. Chem. 275: 32911-32918.

Julenius, K., Thulin, E., Linse, S., and Finn, B.E. 1998. Hydrophobic core substitutions in calbindin D9k: Effects on stability and structure. Biochemistry 37: 8915-8925.

Kharrat, A., Macias, M.J., Gibson, T.J., Nilges, M., and Pastore, A. 1995. Structure of the dsRNA binding domain of E. coli RNase III. EMBO J. 14: 3572-3584.

Koradi, R., Billeter, M., and Wüthrich, K. 1996. MOLMOL: A program for display and analysis of macromolecular structures. J. Mol. Graph. 14: 5155.

Kraulis, P. 1991. MOLSCRIPT: A program to produce both detailed and schematic plots of protein structures. J. Appl. Crystallogr. 24: 946-950.

Krzysiek, R., Lefevre, E.A., Bernard, J., Foussat, A., Galanaud, P., Louache, F., and Richard, Y. 2000. Regulation of CCR6 chemokine receptor expression and responsiveness to macrophage inflammatory protein-3alpha/CCL20 in human B cells. Blood 96: 2338-2345.

Kuszewski, J., Gronenborn, A.M., and Clore, G.M. 1996. Improving the quality of NMR and crystallographic protein structures by means of a conformational database potential derived from structure databases. Protein Sci. 5: 1067-1080.

Laskowski, R.A., MacArthur, M.W., Moss, D.S., and Wright, P.E. 1993. PROCHECK: A program to check the stereochemical quality of protein structures. J. Appl. Crystallogr. 26: 283-291.

Mathews, M., Jia, H.P., Guthmiller, J.M., Losh, G., Graham, S., Johnson, G.K., Tack, B.F., and McCray Jr., P.B. 1999. Production of beta-defensin antimicrobial peptides by the oral mucosa and salivary glands. Infect. Immun. 67: 2740-2745.

Merritt, E.A. and Murphy, M.E.P. 1994. Raster3D version 2.0-A program for photorealistic molecular graphics. Acta Cryst. D50: 869-873.

Nilges, M. 1993. A calculation strategy for the structure determination of symmetric dimers by ${ }^{1}$ H NMR. Proteins 17: 297-309.

Nilges, M., Gronenborn, A.M., Brünger, A.T., and Clore, G.M. 1988. Determination of three-dimensional structures of proteins by simulated annealing with interproton distance restraints. Application to crambin, potato carboxypeptidase inhibitor and barley serine proteinase inhibitor 2. Protein Eng. 2: 27-38.

O’Neil, D.A., Cole, S.P., Martin-Porter, E., Housley, M.P., Liu, L., Ganz, T., and Kagnoff, M.F. 2000. Regulation of human beta-defensins by gastric 
epithelial cells in response to infection with Helicobacter pylori or stimulation with interleukin-1. Infect. Immun. 68: 5412-5415.

Perez-Canadillas, J.M., Zaballos, A., Gutierrez, J., Varona, R., Roncal, F., Albar, J.P., Marquez, G., Bruix, M. 2001. NMR solution structure of murine CCL20/MIP-3 $\alpha$, a chemokine that specifically chemoattracts immature dendritic cells and lymphocytes through its highly specific interaction with the $\beta$-chemokine receptor CCR6. J. Biol. Chem. (in press). (The manuscript is available at http://www.jbc.org/cgi/reprint/M103121200v1.)

Sahasrabudhe, K.S., Kimball, J.R., Morton, T.H., Weinberg, A., and Dale, B.A. 2000. Expression of the antimicrobial peptide, human beta-defensin 1, in duct cells of minor salivary glands and detection in saliva. J. Dent. Res. 79: $1669-1674$

Sawai, M.V., Jia, H.P., Liu, L., Aseyev, V., Wiencek, J.M., McCray, P.B., Ganz, T., Kearney, W.R., and Tack, B.F. 2001. The NMR structure of human $\beta$-defensin-2 reveals a novel $\alpha$-helical segment. Biochemistry 40: 3810 3816.

Sayle, R. 1995. RasMol V2.6 molecular visualisation programme. Glaxo Wellcome Research and Development, Stevevage, Hertfordshire, UK.

Schröder, J.M. and Harder, J. 1999. Human beta-defensin-2. Int. J. Biochem. Cell. Biol. 31: 645-651.

Selsted, M.E. and Harwig, S.S. 1989. Determination of the disulfide array in the human defensin HNP-2. A covalently cyclized peptide. J. Biol. Chem. 264: 4003-4007.

Selsted, M.E., Brown, D.M., DeLange, R.J., and Lehrer, R.I. 1983. Primary structures of MCP-1 and MCP-2, natural peptide antibiotics of rabbit lung macrophages. J. Biol. Chem. 258: 14485-14489.

Selsted, M.E., Harwig, S.S., Ganz, T., Schilling, J.W., and Lehrer, R.I. 1985. Primary structures of three human neutrophil defensins. J. Clin. Invest. 76: $1436-1439$.
Selsted, M.E., Tang, Y.Q., Morris, W.L., McGuire, P.A., Novotny, M.J., Smith, W., Henschen, A.H., and Cullor, J.S. 1993. Purification, primary structures, and antibacterial activities of beta-defensins, a new family of antimicrobial peptides from bovine neutrophils. J. Biol. Chem. 268: 6641-6648.

Tang, Y.Q. and Selsted, M.E. 1993. Characterization of the disulfide motif in BNBD-12, an antimicrobial beta-defensin peptide from bovine neutrophils. J. Biol. Chem. 268: 6649-6653.

Torres, A.M., Wang, X., Fletcher, J.I., Alewood, D., Alewood, P.F., Smith, R., Simpson, R.J., Nicholson, G.M., Sutherland, S.K., Gallagher, C.H., King, G.F., and Kuchel, P.W. 1999. Solution structure of a defensin-like peptide from platypus venom. Biochem. J. 341: 785-794.

Torres, A.M., de Plater, G.M., Doverskog, M., Birinyi-Strachan, L.C., Nicholson, G.M., Gallagher, C.H., and Kuchel, P.W. 2000. Defensin-like peptide-2 from platypus venom: member of a class of peptides with a distinct structural fold. Biochem. J. 348: 649-656.

Vaara, M. 1992. Agents that increase the permeability of the outer membrane. Microbiol. Rev. 56: 395-411.

van Wetering, S., Sterk, P.J., Rabe, K.F., and Hiemstra, P.S. 1999. Defensins: key players or bystanders in infection, injury, and repair in the lung? $J$. Allergy Clin. Immunol. 104: 1131-1138.

Wüthrich, K. 1986. NMR of proteins and nucleic acids, Wiley, New York

Yang, D., Chertov, O., Bykovskaia, S.N., Chen, Q., Buffo, M.J., Shogan, J., Anderson, M., Schröder, J.M., Wang, J.M., Howard, O.M., and Oppenheim, J.J. 1999. Beta-defensins: linking innate and adaptive immunity through dendritic and T cell CCR6. Science 286: 525-528.

Zimmermann, G.R., Legault, P., Selsted, M.E., and Pardi A. 1995. Solution structure of bovine neutrophil beta-defensin-12: the peptide fold of the beta-defensins is identical to that of the classical defensins. Biochemistry 34: $13663-13671$. 